Meta

Journal des traducteurs

Translators' Journal

\title{
Que pense-t-on de l'adaptation publicitaire en Belgique et en Suisse?
}

\section{Roger Boivineau}

Volume 17, numéro 1, mars 1972

L'adaptation publicitaire

URI : https://id.erudit.org/iderudit/002098ar

DOI : https://doi.org/10.7202/002098ar

Aller au sommaire du numéro

Éditeur(s)

Les Presses de l'Université de Montréal

ISSN

0026-0452 (imprimé)

1492-1421 (numérique)

Découvrir la revue

Citer cet article

Boivineau, R. (1972). Que pense-t-on de l'adaptation publicitaire en Belgique et en Suisse? Meta, 17(1), 47-51. https://doi.org/10.7202/002098ar d'utilisation que vous pouvez consulter en ligne.

https://apropos.erudit.org/fr/usagers/politique-dutilisation/ 


\section{Que pense-t-on de l'adaptation publicitaire en Belgique et en Suisse?}

L'adaptation des textes publicitaires n'est pas un phénomène particulier au Canada. On la pratique aussi, naturellement, dans d'autres pays bilingues ou multilingues. C'est pourquoi il nous a semblé intéressant d'interroger à ce sujet quelques publicitaires de pays partiellement francophones, la Belgique et la Suisse. Leurs réponses mettent en évidence la constance des problèmes qui se posent à l'adaptateur, quelles que soient la langue de départ et la langue d'arrivée, et quel que soit le pays.

\section{LA SITUATION EN BELGIQUE}

M. A. H. van Frachen, de l'agence J. Walter Thompson, à Bruxelles, décrit ainsi la situation linguistique en Belgique (ses observations permettront de faire quelques comparaisons intéressantes avec la situation au Canada) :

1. Contexte social. - Depuis l'indépendance de la Belgique (1830) jusqu'aux environs de 1935, la langue française était l'apanage des gens instruits et économiquement forts. La langue néerlandaise (en Belgique on dit généralement : le flamand) était celle des petites gens du nord du pays. Les petites gens du sud de la Belgique parlaient un patois dérivé du français (assez analogue au parler picard des provinces françaises voisines). Dans l'est du pays, c'est un patois allemand qui était utilisé dans la vie courante, l'enseignement étant assuré en français.

2. Contexte économique. - Depuis la fin de la Première Guerre mondiale, mais plus encore depuis la fin de la seconde, le nord du pays (fondamentalement de langue flamande) a connu un développement économique, commercial et industriel très rapide, tandis que le sud perdait beaucoup d'importance et que la capitale, Bruxelles, située au centre (et à énorme majorité francophone : de l'ordre de 80 à $90 \%$ ) voyait se concentrer sur son territoire l'industrie des services, les administrations publiques et privées, tant belges qu'étrangères.

3. Contexte politique. - Le mouvement culturel flamand a pris beaucoup d'importance depuis 1945 et a forcé les autorités publiques et privées à utiliser 
le flamand pour toutes les communications s'adressant à la population flamande ; il a imposé le flamand aux firmes privées situées dans le nord du pays et tend continuellement à établir — par réaction contre les excès francophones des débuts de l'indépendance - un déséquilibre linguistique au détriment du français.

Question 1 : D'une façon générale, quelle est, dans votre pays, la langue dominante de la publicité, et pour quelles raisons?

Les réponses reçues à cette question permettent de penser que le français et le néerlandais sont à peu près à égalité. On estime par ailleurs qu'environ $5 \%$ de la publicité diffusée en Belgique l'est en anglais. (Il existe une assez forte colonie anglophone en Belgique : Otan, quartier général des forces américaines en Europe, sociétés américaines et anglaises, etc.)

Question 2 : Dans votre agence, la publicité destinée aux francophones est-elle toujours rédigée directement en français?

Une seule agence déclare que toute sa publicité est toujours directement rédigée en français. Il a été impossible de savoir de façon précise quelle est la proportion d'annonces qui sont adaptées, en particulier du flamand (30\%, selon un de nos correspondants). Certains annonceurs flamands demandent que leurs campagnes soient créées dans leur langue et adaptées en français. Des campagnes " européennes» ou «mondiales» sont créées en anglais et adaptées en français et en flamand.

Par ailleurs, une proportion importante d'annonces rédigées en français sont adaptées en flamand.

\section{LA SITTUATION EN SUISSE}

Question 1 : En Suisse, la langue dominante de la publicité est l'allemand; elle est parlée par la plus grande partie de la population ( $70 \%$ environ). La suprématie de l'allemand s'explique également par le fait que les agences les plus importantes sur le plan national sont situées en Suisse alémanique. Selon un de nos correspondants, la répartition linguistique de la publicité serait de : 5/9 pour l'allemand, 3/9 pour le français, 1/9 pour l'italien.

QuEsTION 2 : Trois de nos correspondants sur quatre nous disent que la publicité destinée aux francophones n'est pas toujours rédigée directement en français. En général, les adaptations de l'allemand en français sont plus fréquentes que celles faites dans le sens inverse, mais la publicité en français est adaptée en allemand, et surtout en italien.

On fera le rapprochement avec le Canada où l'adaptation dans le sens français-anglais est rarissime.

\section{RÉPONSES À DES QUESTIONS D’ORDRE GÉNÉRAL}

QUESTION 3 : Lorsque la publicité est adaptée d'une autre langue, s'agit-il la plupart du temps : d'une traduction pure et simple, d'une adaptation assez large $d u$ texte original, ou d'une adaptation empruntant des éléments du texte original et les combinant avec des éléments conçus en français?

Pour nos confrères belges, la deuxième solution (adaptation assez large) est la plus fréquente. C'est également le cas en Suisse, mais les publicitaires suisses signalent que la traduction pure et simple se pratique également. 
QUESTION 4 : Selon vous, l'adaptation publicitaire est-elle une solution acceptable?

«Tout dépend de ce qu'on entend par adaptation. Il faut distinguer la lettre de l'esprit. Tout texte peut être adapté à condition que l'adaptateur respecte la psychologie du lecteur. Il n'y a pas seulement des différences de langues mais d'ethnies; les façons de percevoir, de réagir peuvent changer légèrement selon que le lecteur est francophone ou néerlandophone» (Guy Brun d'Aubignosc, Publicontrol Benton \& Bowles, Bruxelles).

«Dans notre cas oui, vu les nombreux points communs que possèdent tous les Belges, malgré les tensions linguistiques existant entre eux» (A. H. van Frachen, J. Walter Thompson, Bruxelles).

«L'adaptation publicitaire est une solution moyenne qui est déjà une amélioration par rapport à la traduction pure et simple, mais elle est certainement moins bonne que la conception originale dans les deux langues nationales» ( $\mathrm{J}$. Vanden Schrieck, World Publicité, Bruxelles).

«L'adaptation est la seule solution acceptable puisqu'une traduction pure et simple est toujours mauvaise. D'autre part, l'adaptation est nécessaire, puisque le client veut souvent que le message conçu dans une langue soit transmis tel quel dans l'autre langue ${ }^{1} \gg$ (Denyse Lamprière, Schoop-Propagande, Genève).

"Comment veut-on qu'il en soit autrement? Le devoir de l'entreprise étant de se créer une image de marque et de promouvoir des produits ou des services bien déterminés, il va de soi que la politique publicitaire se fonde sur des motivations quasi universellement acceptées. La fameuse réponse «this is wonderful... but not for our country » appartient au sottisier classique de la publicité internationale» (G. Caspari, Georges Caspari Advertising Agency, Genève).

«Solution acceptable, oui. Pour des raisons budgétaires et de création d'une image multinationale » (Thomas Weinstock, ea-Consultas, Zürich).

«Oui, dans la mesure où, si nécessaire, elle peut être très libre. Il faut en outre que la conception de base (d'une campagne publicitaire par exemple) ait été faite avec le rédacteur de la langue étrangère à adapter (ou qu'elle puisse être discutée avec lui) »(Louis Chevallier, Reinhardt Publicité, Genève).

QUESTION 5 : Quels sont, d'après votre expérience, les principaux obstacles et difficultés rencontrés dans l'adaptation de textes publicitaires?

« $1^{\circ}$ Chaque langue a son génie : une litote, un mot humoristique peuvent être inadaptables; à la limite on peut provoquer l'effet contraire à celui recherché ; $2^{\circ}$ Souvent, il n'y a pas parfaite équivalence entre les mots qui, selon la langue, recouvrent des réalités différentes ; $3^{\circ}$ Problèmes de longueur du texte : plus long en néerlandais qu'en français ou le contraire» (G. Brun d'Aubignosc).

«L'adaptateur doit bien comprendre le problème. Il doit comprendre le processus de pensée qu'a suivi le créateur. Il doit connaître la langue de création de façon suffisante pour ne pas trahir la pensée de l'auteur lors de l'adaptation » (A. H. van Frachen).

«[...] les deux langues (français et néerlandais) ayant leurs idiomes propres, une traduction ou une adaptation risque de provoquer des interprétations contraires

1. Il est réconfortant de constater que le problème évoqué ici par notre correspondante ne se présente pas uniquement au Québec!N.D.L.R. 
à la conception fondamentale de l'une ou l'autre des mentalités des deux régions linguistiques »(J. Vanden Schriek).

«Le principal obstacle vient des jeux de mots souvent intraduisibles. Ils sont d'autant plus complexes qu'ils figurent dans un titre et que toute l'annonce, illustration comprise, en dépend. D'autre part, le «génie de la langue » joue un grand rôle, car la façon de considérer tel ou tel fait est souvent différente d'une partie à l'autre du pays » (D. Lamprière).

«En premier lieu, le client. Dans notre pays, où il est censé parler au moins trois langues (l'allemand, l'anglais et le français), il est volontiers enclin à se prendre pour l'égal d'un traducteur professionnel. En second lieu - mais seulement pour les produits de consommation courante, alimentation, etc. - le mode de vie, les habitudes physiologiques, etc., qui sont fondamentalement différentes en Suisse alémanique, romande ou italienne » (G. Caspari).

"L'impossibilité, quelquefois, de rendre l'impact des langues coagulantes (allemand) ou la concision de l'anglais. Dans ce dernier cas, le problème peut être dans la vigueur des titres (plus de mots outils en français) ou la longueur des textes (environ $1 / 3$ de plus en français). La nécessité, pour l'auteur du texte de base, d'éviter tout jeu de mots ou locution intraduisibles (lorsqu'ils sont un élément important du message publicitaire) » (L. Chevallier).

QuESTION 6: Le public peut-il distinguer une annonce créée dans sa langue d'une annonce adaptée?

"Quand l'annonce est bien adaptée, impossible de savoir si elle a été conçue dans une autre langue » (G. Brun d'Aubignosc).

"Oui, s'il y retrouve des mots ou des lambeaux de phrases traduits sans nuances 》 (A. H. van Frachen).

"Oui, très aisément, et c'est la raison pour laquelle il y a lieu de l'éviter 》 (J. Vanden Schrieck).

"Malheureusement oui. Mais bien davantage dans le sens du texte allemand traduit en français qu'à l'inverse. Le Français et le Suisse français sont maladivement attachés aux nuances de leur langue maternelle - et par conséquent plus respectueux de la langue de l'«autre» (G. Caspari).

"Oui, si l'annonce n'a pas été créée dans les conditions citées à la réponse à la question 4 (autrement dit, si elle a été plus traduite qu'adaptée). Sinon, tout dépend de la qualité du rédacteur et de la liberté dont il dispose »(L. Chevallier).

«Non, s'il s'agit de sujets techniques ou si l'adaptation est suffisamment libre. Oui, si la transposition n'est qu'incomplète » (D. Lamprière).

«En général non. Surtout si la traduction est assez loin du texte original » (T. Weinstock).

Question 7 : D'une façon générale, le rendement de la publicité adaptée est-il inférieur, égal ou supérieur à celui de la publicité originale? (Votre réponse s'appuie-t-elle sur des sondages $^{2}$ ?)

«Nous avons envie de répondre que le rendement est inférieur s'il s'agit d'adaptation du français à l'allemand ou vice versa. En revanche, une publicité

2. Aucune des réponses reçues ne s'appuie sur des sondages. Quatre de nos correspondants ne se sont pas prononcés au sujet du rendement. N.D.L.R. 
bien pensée et bien développée en français se trouve souvent valorisée en anglais » (G. Caspari).

"Appréciation empirique : rendement égal si l'adaptation est bonne et si les autres données (motivations, donc conception) sont correctement adaptées au public qui lira le texte $\gg$ (L. Chevallier).

«Égal pour les annonces, inférieur pour la publicité directe »(D. Lamprière).

Propos recueillis par

ROGER BOIVINEAU 